\title{
MeCP2 modulates gene expression pathways in astrocytes
}

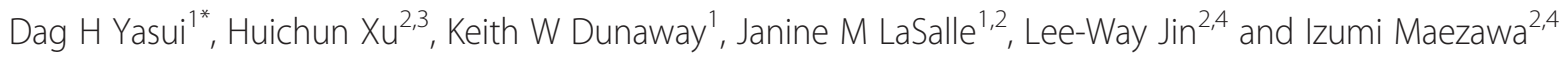

\begin{abstract}
Background: Mutations in MECP2 encoding methyl-CpG-binding protein 2 (MeCP2) cause the X-linked neurodevelopmental disorder Rett syndrome. Rett syndrome patients exhibit neurological symptoms that include irregular breathing, impaired mobility, stereotypic hand movements, and loss of speech. MeCP2 protein epigenetically modulates gene expression through genome-wide binding to methylated $\mathrm{CpG}$ dinucleotides. While neurons have the highest level of MeCP2 expression, astrocytes and other cell types also express detectable levels of MeCP2. Recent studies suggest that astrocytes likely control the progression of Rett syndrome. Thus, the object of these studies was to identify gene targets that are affected by loss of MeCP2 binding in astrocytes.

Methods: To identify gene targets of MeCP2 in astrocytes, combined approaches of expression microarray and chromatin immunoprecipitation of MeCP2 followed by sequencing (ChIP-seq) were compared between wild-type and MeCP2-deficient astrocytes. MeCP2 gene targets were compared with genes in the top 10\% of MeCP2 binding levels in gene windows either within $2 \mathrm{~kb}$ upstream of the transcription start site, or the 'gene body' that extended from transcription start to end site, or $2 \mathrm{~kb}$ downstream of the transcription end site.

Results: A total of 118 gene transcripts surpassed the highly significant threshold $(P<0.005$, fold change $>1.2)$ in expression microarray analysis from triplicate cultures. The top 10\% of genes with the highest levels of MeCP2 binding were identified in two independent ChIP-seq experiments. Together this integrated, genome-wide screen for MeCP2 target genes provided an overlapping list of 19 high-confidence MeCP2-responsive gene transcripts in astrocytes. Validation of candidate target gene transcripts by RT-PCR revealed that expression of Apoc2, Cdon, Csrp and Nrep were consistently responsive to MeCP2 deficiency in astrocytes.
\end{abstract}

Conclusions: The first MeCP2 ChIP-seq and gene expression microarray analysis in astrocytes reveals a set of potential MeCP2 target genes that may contribute to normal astrocyte signaling, cell division and neuronal support functions, the loss of which may contribute to the Rett syndrome phenotype.

Keywords: MeCP2, Epigenetics, Astrocytes, Rett syndrome, ChIP-seq, Transcription

\section{Background}

Rett syndrome (RTT) is an X-linked neurologic disorder representing one of the most frequent causes of severe mental retardation in females. RTT infants develop normally until six to eighteen months of age but then develop progressive loss of neurodevelopmental milestones [1]. Clinical features include deceleration of brain growth, loss of motor skills including purposeful hand movements, absence of speech, seizures, and respiratory irregularity [2]. Mutations in X-linked $M E C P 2$ encoding

\footnotetext{
* Correspondence: dhyasui@ucdavis.edu

'Department of Medical Microbiology and Immunology, UC Davis Genome Center, University of California Davis, 1 Shields Avenue, Davis, CA 95616, USA Full list of author information is available at the end of the article
}

methyl-CpG-binding protein $2(\mathrm{MeCP} 2)$ are responsible for most cases of RTT [3], although mutations in CDKL5 and FOXG1 were recently identified in $M E C P 2$ mutationnegative individuals with RTT features [4-6].

$\mathrm{MeCP} 2$ is one member of a family of DNA-binding proteins that was originally hypothesized to silence gene transcription by binding to methylated $\mathrm{CpG}$ dinucleotides in promoters [7]. This model predicts that MeCP2 deficiency should result in activation of normally repressed genes. However, early genome-wide expression profiling studies revealed that only a few genes were significantly upregulated and surprisingly, some genes appeared to be repressed even in MECP2-knockout mice displaying overt disease symptoms [8]. In addition, an integrated, genome-

\section{Biomed Central}


scale chromatin immunoprecipitation (ChIP) and expression microarray analysis revealed that $63 \%$ of active promoters were bound by MeCP2 and of these only $6 \%$ were highly methylated [9]. Subsequent genome-wide expression analyses of hypothalamus indicate that MeCP2 primarily activates but can also represses transcription [10]. More recent analyses of MeCP2 binding by ChIPsequencing (ChIP-seq) have shown binding at nearhistone occupancy levels throughout the neuronal genome $[11,12]$. Thus, MeCP2 may function as a genome-wide, transcriptional modulator similar to histone modifiers of chromatin. MeCP2 has also been shown to act as a chromatin organizer both in vivo [13,14] and in vitro [15]. In addition, MeCP2 appears to have a role in the regulation of splicing [16]. In summary, MeCP2 appears to have multiple, genome-wide, gene regulatory functions.

As the symptoms of RTT syndrome are primarily neurological, MeCP2 function was originally hypothesized to be restricted to neurons $[17,18]$. It appears that MeCP2 binding to DNA and subsequent transcriptional modulation is a dynamic process and dependent on external signals such as $\mathrm{Ca}^{2+}[19,20]$. One example of transcriptional regulation is provided by Greenberg and colleagues [19,20]. Their studies suggest that neuronal membrane depolarization triggers calcium-dependent phosphorylation at serine 421 and release of $\mathrm{MeCP} 2$ from the $B d n f$ promoter III, thereby facilitating transcription [20]. These results indicate that $\mathrm{MeCP} 2$ plays a key role in the transcription of neuronal activity-dependent gene regulation.

Although MeCP2 is important for neuronal function, many studies suggest that the function of other cell types, particularly astrocytes, is impaired by $\mathrm{MeCP} 2$ defects. Although MeCP2 levels are roughly five-fold lower in astrocytes than in neurons $[11,21]$, recent studies suggest that loss of MeCP2 in astrocytes contributes to Rett-like symptoms and restoration of MeCP2 can rescue some of these defects [22]. In contrast to neuronal studies [19], levels of $B d n f$ transcript and protein were found to be upregulated in Mecp2-null astrocytes [21]. These results may reflect different MeCP2 gene regulatory roles in astrocytes and neurons that are indicative of different functions of these cell types in the brain. Genome-wide identification of MeCP2 targets in astrocytes is therefore likely to identify cell-type-specific genes necessary for normal astrocyte function, and subsequently normal brain function.

Prior attempts to identify MeCP2-targeted genes by genome-wide expression profiling were based on the assumption that $\mathrm{MeCP} 2$ acts as a transcriptional repressor in neurons. However, the list of identified MeCP2 target genes across different tissues and cell types and even different studies is tremendously variable with essentially non-overlapping gene lists [8,10,23-27]. The results of these previous studies are confounded by the possibility that MeCP2 has different functions in different cell types and the fact that MeCP2 varies by several fold in different brain cell types [21]. Here, we take into account these new findings and extend integrated MeCP2 ChIP and expression profiling analyses to identify MeCP2 target genes that may contribute to astrocytic abnormalities and neurological deficits in RTT.

\section{Methods}

\section{Mecp2/MeCP2-deficient and control astrocyte cultures}

Mecp $2^{\mathrm{tm} 1.1 \mathrm{Bird} /+}$ mice developed by Guy et al. [17] were obtained from The Jackson Laboratory, Bar Harbor, ME, USA. Females heterozygous for the mutation were mated with C57BL/6 J wild-type males (The Jackson Laboratory). Pups were genotyped using genomic DNA isolated from tail snips to determine Mecp 2 allele type according to the protocol designed by the original investigators [17]. Gender was determined using primers for the Sry gene on Y chromosome, which were $5^{\prime}$-TGG GAC TGG TGA CAA TTG TC-3' and 5'-GAG TAC AGG TGT GCA GCT CT-3'. The University of California Davis Institutional Animal Care and Use Committee approved all animal protocols. Primary astrocyte cultures were prepared from postnatal day 1 (P1) mouse cerebral cortex according to previously described methods [28] for expression profiling and $\mathrm{MeCP} 2 \mathrm{ChIP}$-seq. In most experiments, two to four weeks in vitro, confluent astrocyte cultures were used, which showed no differences in cell number, total RNA and protein levels between different Mecp2 genotypes. In both expression profiling and ChIP-seq experiments astrocytes were combined or pooled from multiple wild-type and MeCP2-deficient pups to provide sufficient material for experimental procedures. ChIP validation primers are listed in Table S3 in Additional file 1.

\section{Expression microarray hybridization}

Total RNA was isolated by homogenization of cell cultures and processing using TRIzol reagent (Invitrogen, Carlsbad, CA, USA) according to the manufacturer's instruction. Purified RNA was resuspended in RNasefree water and stored at $-70^{\circ} \mathrm{C}$. RNA purity and integrity were assessed using a spectrophotometer (Nanodrop Technologies, Wilmington, DE, USA) and bioanalyzer analyses (Agilent Technologies Inc., Palo Alto, CA, USA). Five $\mu g s$ of each total RNA was labeled, hybridized, and scanned according to the Affymetrix technical manual (Affymetrix GeneChip ${ }^{\mathrm{TM}}$ Expression Analysis Technical Manual 701023, Rev. 4) with whole genome 'GeneChip ${ }^{\text {TM }}$ Mouse Expression 430 2.0' microarrays (Affymetrix, Santa Clara, CA, USA). Only samples with an A260/A280 absorbance ratio greater than 1.9 and a 28S/ $18 \mathrm{~S}$ rRNA ratio greater than 1.5 were analyzed. The GeneChip $^{\text {TM }}$ Mouse Expression 4302.0 microarray contains 
45,000 probe sets representing over 34,000 well-substantiated mouse gene transcripts.

\section{Expression microarray data analysis}

Raw image signals from the scanning were transformed into. CEL files. To determine if a transcript was present or absent, the probe was then analyzed using the MAS5 algorithm in Genespring GX 10 software (Silicon Genetics, Redwood City, CA, USA). On the mouse 4302.0 array, a transcript is represented as a probe set. Each probe set is made up of a set of probe pairs including perfect match (PM) and mismatch (MM) probe cells. Mismatch probes are utilized to adjust the perfect match intensity. Each probe pair in a probe set has a potential influence in determining whether the measured transcript is detected (present or marginally present) or not detected (absent). The Wilcoxon rank test is used to calculate a $P$ value and detection call for each probe set. Two prefiltered gene lists were then generated to form the basis for subsequent statistical analysis, which will be described below.

The first prefiltered gene list will be used to determine genes upregulated in MeCP2-difficient samples compared to wild-type controls. For this purpose, only those genes showing 'present' or 'marginally present' in at least two of the three Mecp2-deficient samples were included. This represents about $55.1 \%$ (24,863 probe sets) of all the transcripts measured (45,101 probe sets). Similarly, to determine the downregulated genes in MeCP2deficient samples compared to wild-type controls, only including those genes 'present' or 'marginally present' in at least two of the three wild-type control samples, a second prefiltered gene list was generated. This second gene list consisted of about $55.8 \%$ (24,863 probe sets) of all the transcripts measured.

Expression intensity for each probe set was then calculated with the guanine cytosine robust multiarray average (GCRMA) algorithm from the raw image signals (robust multichip average with GC-content background correction algorithm, http://www.bioconductor.org). The GCRMA algorithm involves background correction, quartile normalization, summarization of the probe-set value into gene-level expression measurements [29]. After GCRMA normalization, the expression data was log 2-transformed. The expression data was then analyzed using statistical tools in Genespring GX 10 software.

To identify differentially regulated genes between MeCP2-deficient samples and wild-type controls, a Student's unpaired $t$ test with Benjamin-Hochberg false discovery rate (FDR) control (0.05) or no FDR control was conducted based on the two prefiltered gene lists generated under the MAS5 algorithm to identify upregulated genes and downregulated genes, respectively [30]. In the case without FDR control, a stringent $P$ value less than 0.005 was used. Then, a minimum 1.2 fold or 1.5 fold cut off was applied to further minimize false positive findings (Table S2 in Additional file 1). The differentially regulated genes determined by above methods were then subjected to function annotation analysis in the Database for Annotation, Visualization and Integrated Discovery (DAVID) 2008 (http://www.david.abcc.ncifcrf.gov) [31,32].

\section{ChIP sequencing and alignment}

MeCP2 ChIP was performed on chromatin prepared from pooled wild-type (WT) and pooled MeCP2-deficient cultured astrocytes, (referred to as MeCP2 and $\mathrm{KO}$ ) as described previously (Yasui et al. [9]). Libraries were prepared from ChIP-isolated fragments using a protocol and reagents from Illumina (Illumina, San Diego, CA, USA). Reference or input DNA was prepared in parallel from crosslinked chromatin isolated from wild-type astrocytes. High throughput sequencing of MeCP2 ChIP and control libraries was performed using the Illumina-Solexa sequencing platform for single end, 76 base long reads. MeCP2 ChIP library reads were then aligned to the mouse consensus genome ( $\mathrm{mm} 9$ build) using bowtie (version 0.12.7) with the following parameters: $-\mathrm{M}=1,-\mathrm{k}=1,-$ strata, - best. This yielded reads: input 17,577,122 aligned (15,385,724 unique and 2,191,398 non-unique); MeCP2 47,305,937 aligned (41,520,644 unique and 5,785,293 non-unique); and KO-MeCP2 16,779,645 aligned (14,662,294 unique and 2,117,351 non-unique).

To validate specific MeCP2 target regions identified by ChIP-seq, chromatin from independently generated in vitro astrocyte cultures derived from pooled mouse brains were prepared. Astrocyte chromatin was sonicated to approximately 200 to $400 \mathrm{bp}$ using a Bioruptor 300 (Diagenode, Sparta, NJ, USA). Chromatin fragments and subjected to ChIP with anti-MeCP2 and control IgY antibodies as described in Yasui et al. [9]. Real-time, quantitative PCR (qPCR) was used to assess enrichment of $\mathrm{MeCP}$-bound target regions.

\section{ChIP-seq analysis}

MeCP2 ChIP-seq data was first analyzed using established ChIP-seq algorithms including Sole-Search [33] ChromaSig [34] and MACS [35]. However, these algorithms which were designed to identify distinct peaks of transcription factor binding seemed unsuited to the subtle domain patterns of MeCP2 binding in astrocytes. Therefore, a novel ChIP-seq analysis method was developed for these studies. For this analysis, the number of reads per kilobase of element assayed per million mapped reads (RPKM) for MeCP2 astrocyte ChIP-seq and input were calculated for three regions per gene: $2000 \mathrm{bp}$ up- and down-stream of the transcription start site (TSS), 2000 bp up- and down-stream of the transcription end site (TES), and within the gene itself, which included both exons and introns (gene body). Input RPKM was 
subtracted from MeCP2 ChIP RPKM in order to normalize for genomic sequencing bias. Normalized RPKMs were ranked and the top 10\% of genes were used in each of the three categories to compile the MeCP2 ChIP-seq lists TSS, gene body, and TES. The 118 astrocyte genes determined to be differentially expressed by microarray analysis were compared to genes on each of the MeCP2 ChIP-seq lists to produce a list of 19 potential target genes. A select group of genes from each list were selected for RT-PCR analysis validation.

\section{RT-PCR analysis}

For confirmation of Affymetrix expression microarray results, RT-PCR analysis was performed as described previously [14]. Briefly, total RNA was isolated using TRIzol ${ }^{\mathrm{TM}}$ Reagent (Invitrogen, Carlsbad, CA, USA). To remove residual DNA, total RNA was treated with DNaseI (New England Biolabs, Ipswich, MA, USA) according to manufacturer's instructions. Single-stranded complimentary DNA (cDNA) was synthesized using Quantitect Reverse Transcription Kit (Qiagen, Valencia, CA, USA). Genespecific primers (Table S1 in Additional file 1) were designed to cross an intron or span intron/exon boundaries to further limit the effect of potential genomic DNA contamination using Biosearch Technologies Real Time Design software (Biosearch Technologies, Novato, CA, USA) (http://www.biosearchtech.com/realtimedesign).

PCR amplification of CDNA was performed using Express Sybr ${ }^{\mathrm{TM}}$ Green ER Universal Master mix (Invitrogen) on a Mastercycler ${ }^{\mathrm{TM}}$ ep realplex (Eppendorf, Hamburg, Germany). Crossing points were analyzed using realplex software (Eppendorf). For each reaction a well without reverse transcriptase and a well without control cDNA were amplified to evaluate genomic DNA contamination, non-specific product formation or other contamination. All samples were normalized to Gapdh expression using the comparative Ct method (Applied Biosystems, Foster City, CA, USA) to measure fold change relative to a calibrator. Melting curve analysis was also performed to determine homogenous product formation. Pair-wise comparison of cDNA samples was performed using a one-tailed Student's $t$ test where significance is defined as $P<0.05$.

\section{Results}

To identify genes affected by MeCP2 deficiency specifically in astrocytes, expression microarray analysis was performed on RNA extracted from nearly pure (> 95\%) astrocyte cultures derived from Mecp2-deficient and wild-type cortices. First, analysis of hybridized probe intensities of Mecp2 transcripts was performed on microarray data (Figure 1) to confirm that Mecp2 was deficient in mutant astrocyte cultures. Two separate probe sets detected Mecp 2 transcripts in three independent technical replicates from wild-type control astrocytes, while

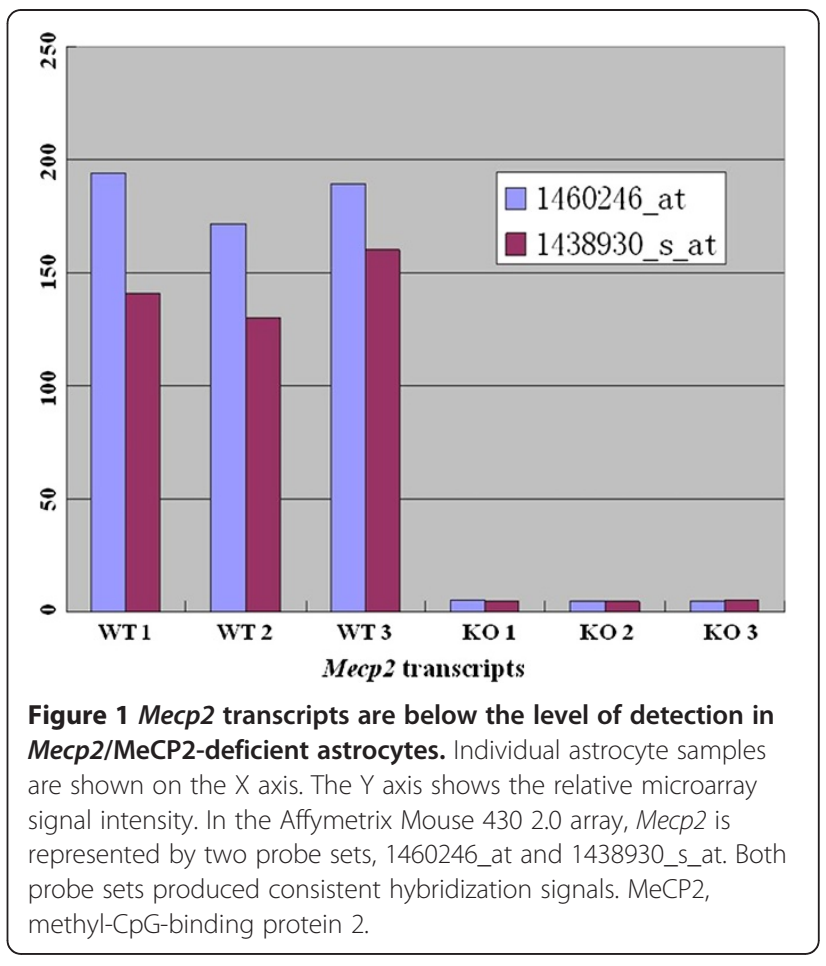

astrocytes from MeCP2-deficient mice gave only background signal levels. These results and subsequent RT-PCR analysis of transcript levels (supplemental) confirms the absence of significant Mecp2 transcripts in genetargeted astrocytes.

After confirmation of Mecp2 transcript ablation in astrocytes, an analysis of differential gene transcripts between Mecp2-deficient and wild-type astrocytes was performed. Stringent analysis of microarray signals revealed that 142 other probes representing 118 genes passed the $P<0.005$ and fold change $>1.2$ differential expression threshold with 55 genes upregulated and 63 downregulated (Table S2 in Additional file 1). DAVID analysis of this conservative, differentially expressed gene list revealed significant $(P<0.05)$ enrichment of genes in the long-term depression signaling pathway included: Crh encoding corticotropin-releasing hormone, Gria1 encoding glutamate receptor, ionotropic AMPA1 and $P p p 2 r 2 b$ encoding protein phosphatase 2; regulatory subunit $\mathrm{B}$ and genes in the cytokine-cytokine receptor interaction pathway including $P d g f b$ encoding platelet-derived growth factor beta, Il13ral encoding interleukin 13 receptor alpha 1 and Osmr encoding oncostatin M receptor. DAVID analysis also revealed that transcripts were significantly dysregulated by loss of MeCP2-encoded factors involved in multiple signaling pathways, including the immediate early genes Jun (Jun proto-oncogene) and Fos (FBJ murine osteosarcoma viral oncogene homolog). Additional dysregulated transcripts that have previously shown to have enriched expression in astrocytes [36] 
were identified in this screen include Ide encoding insulindegrading enzyme, Gabrg1 encoding GABAA receptor gamma 1, and Egr1 encoding early growth response 1 protein. Heat-map hierarchical clustering analysis of the 118 genes revealed consistent gene expression patterns in wild-type astrocytes that were distinct from Mecp2deficient astrocytes (Figure 2), illustrating the reproducibility of the expression microarray data.

To determine MeCP2-bound genes throughout the astrocyte genome, ChIP was performed on a parallel set of cultures, using a custom anti-MeCP2 antibody [9] followed by high throughput sequencing. For these studies, a novel ChIP-seq analysis method was developed (see Methods). Genes were ranked based on normalized averaged levels of MeCP2 binding in three windows: 1) upstream of the transcriptional start site (TSS), 2) within the gene body, or 3) downstream of the transcriptional end site (TES). The top $10 \%$ of MeCP2-bound genes in each of the three categories were compared to the 118 genes identified by expression microarray to identify the most likely target genes. This stringent filtering produced mostly non-overlapping gene lists (Figure 3).

Six genes satisfied the criteria for MeCP2 binding at the TSS and significant gene expression changes in MeCP2-deficient astrocytes (Figure 3A). These genes were Ppp1r32 (protein phosphatase 1, regulatory subunit 32), Aard (alanine and arginine-rich domain-containing protein), Apoc2 (apolipoprotein C-II), Nrep (neuronal regeneration-related protein), Egr1 (early growth response 1) and Irx3 (Iroquois related homeobox 3). Although the classic model of MeCP2 function predicts that binding to $\mathrm{CpG}$ island promoters represses gene expression, Apoc2 was the only gene of this group to show elevated transcripts in the absence of MeCP2. MeCP2 ChIP-seq reads upstream of the TSS of Aard are shown as an example in Figure 4A.

Eight genes with significant expression changes also had high MeCP2 binding levels at gene body in the top $10 \%$ quantile (Figure 3B). These included Hide (highly expressed in immature dendritic cell transcript, Cdon

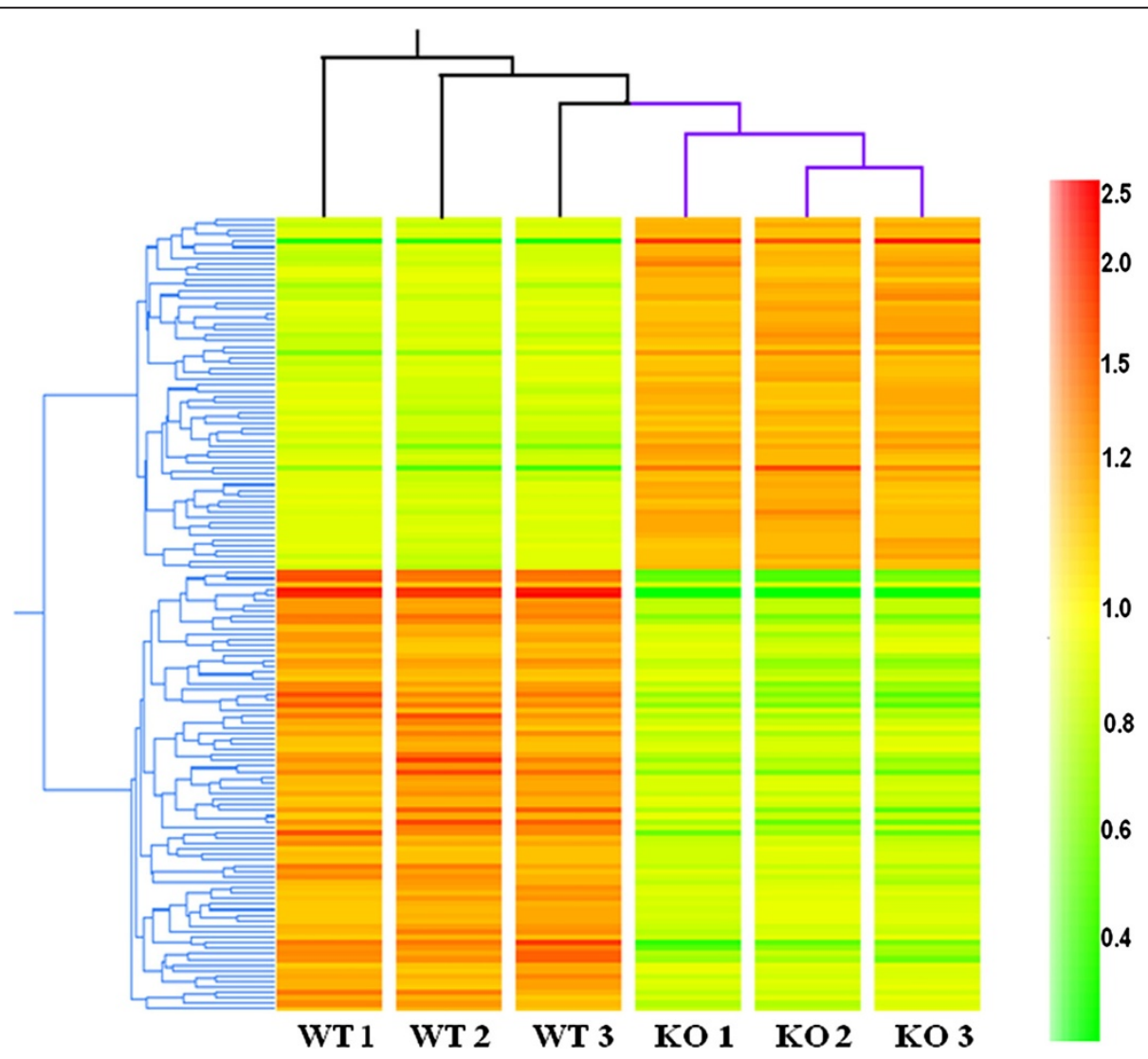

Figure $2 \mathrm{~A}$ heat map showing gene expression levels of 119 transcripts including MeCP2 differentially regulated between astrocytes with Mecp2/MeCP2 deficiency and wild-type astrocytes at $P<0.005$ and $\mathbf{1 . 2}$ fold change threshold. Each column corresponds to individual astrocyte samples (WT: wild-type astrocytes; KO: Mecp2/MeCP2-deficient astrocytes). Each row represents an individual gene. Expression signal intensity was color-coded with red being high and green being low, as shown by the bar to the right. The tree structures to the left and on the top demonstrated similarities between genes and samples, respectively, according to Pearson cluster analysis results. Thus the more similar, the smaller the distance between the tree branches. MeCP2, methyl-CpG-binding protein 2. 


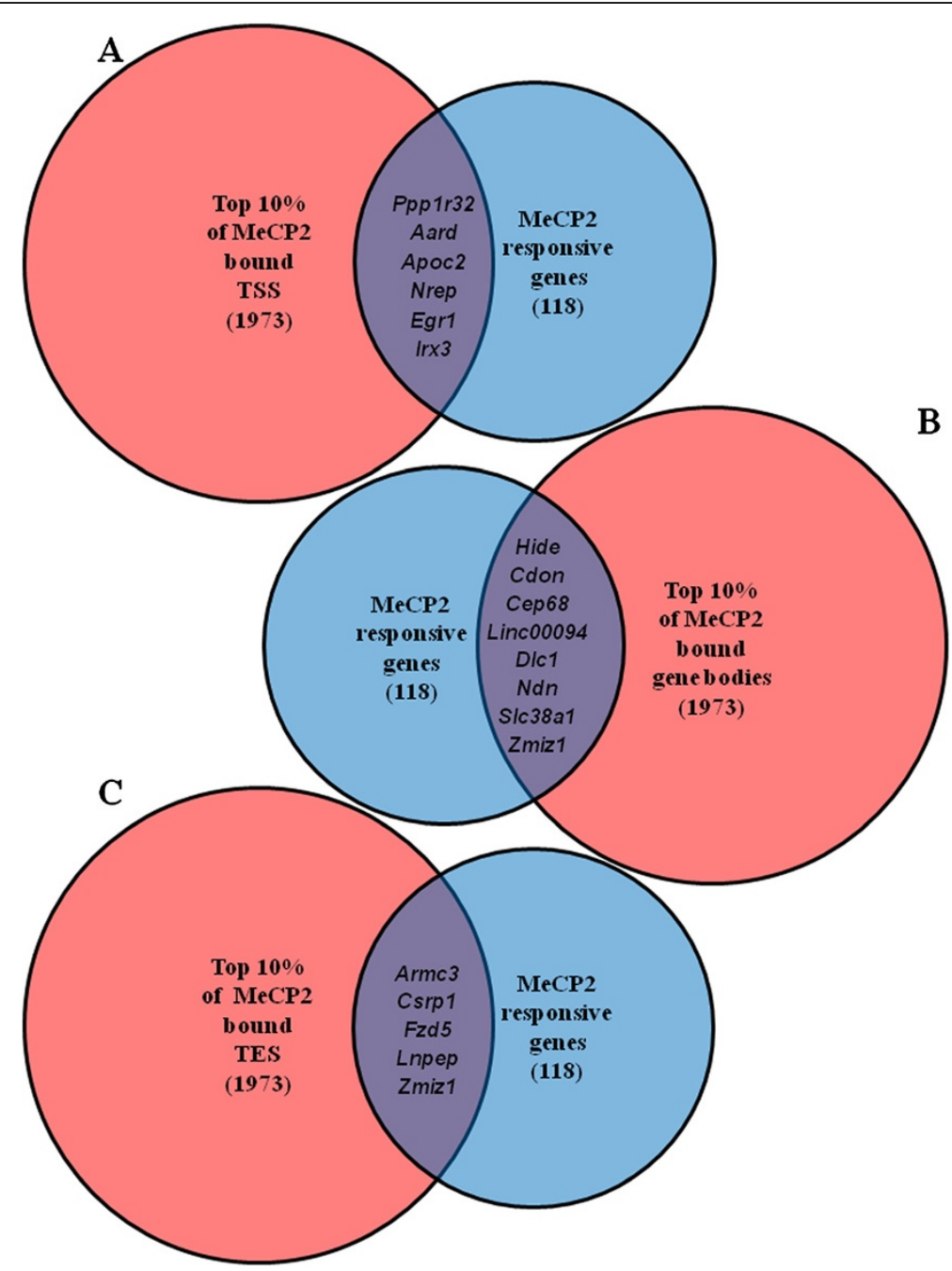

Figure $3 \mathbf{M e C P} 2$ binding is enriched in different regions of modulated genes. (A) Venn diagram comparing the top 10\% of MeCP2-bound TSS with genes (large circle) having the highest significance in differential expression $(P<0.005$, fold change $>1.2)$ in MeCP2-deficient astrocytes (small circle). (B) Top 10\% of MeCP2-bound gene bodies compared to the MeCP2-responsive transcripts. (C) Top 10\% of MeCP2-bound TES compared to MeCP2-responsive transcripts. Zmiz is the only gene transcript to have high levels of MeCP2 binding in more than one region. MeCP2, methyl-CpG-binding protein 2; TES, transcriptional end sites; TTS, transcriptional start sites.

(cell adhesion molecule-related/downregulated by oncogenesis), Cep68 (centrosomal protein of $68 \mathrm{kDa}$ ), Linc00094 (long intergenic non-protein coding RNA 94), Dlc1 (deleted in liver cancer 1), $N d n$ (necdin), Slc38a1 (solute carrier family member 38 , member 1 ) with enriched MeCP2 binding reads shown in Figure 4B and Zmiz1 (zinc finger, MIZ-type containing 1). Linc00094, $N d n$ and Slc38a1 showed reduced expression in MeCP2deficient astrocytes while the rest of the genes had elevated expression compared to wild-type astrocytes.

Also, Armc3 (armadillo repeat containing 3), Csrp1 (cysteine and glycine-rich protein 1), Fzd5 (frizzled homolog 5), Lnpep (leucyl/cystinyl aminopeptidase) and Zmiz1 (zinc finger, MIZ-type containing 1) had above threshold levels of MeCP2 binding at the TES and significantly altered transcript levels (Figure 3C). Interestingly, with the exception of Armc3, which is shown as representative of an MeCP2 bound TES (Figure $4 \mathrm{C}$ ), all the genes with the highest level of MeCP2 binding to the TES had upregulated expression in MeCP2-deficient cells consistent with repression (Table 1). The zinc finger regulator of androgen receptor, Zmizl encoding zinc finger, MIZ-type containing 1 protein had high levels of MeCP2 binding in more than one region.

To further validate MeCP2 ChIP-seq results, quantitative ChIP-PCR (qChIP-PCR) analysis was performed in an independent set of astrocyte cultures. Using the same experimental conditions employed for ChIP-seq, astrocyte chromatin was assayed for MeCP2 binding to representative genes shown in Figure 4. As expected, Aard shows 


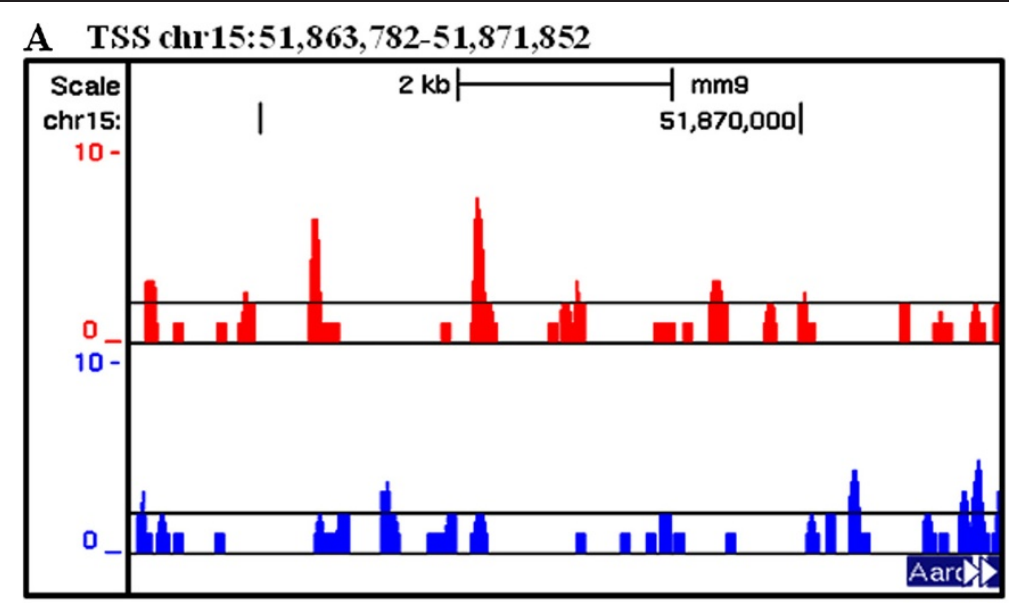

B Gene body chr15:96,418,354-96,468,037

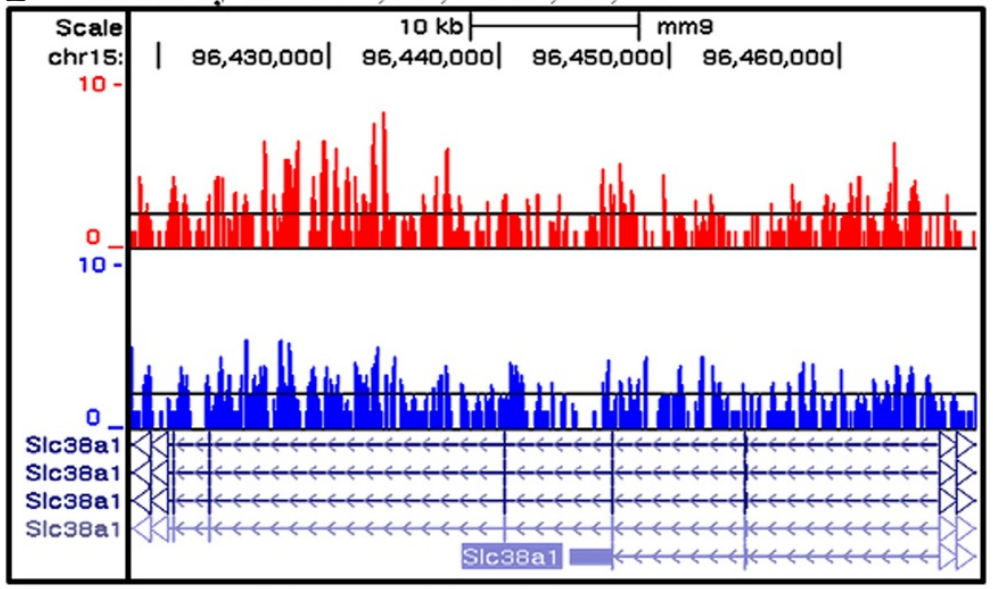

C Chr2:chr2:19,229,234-19,241,224

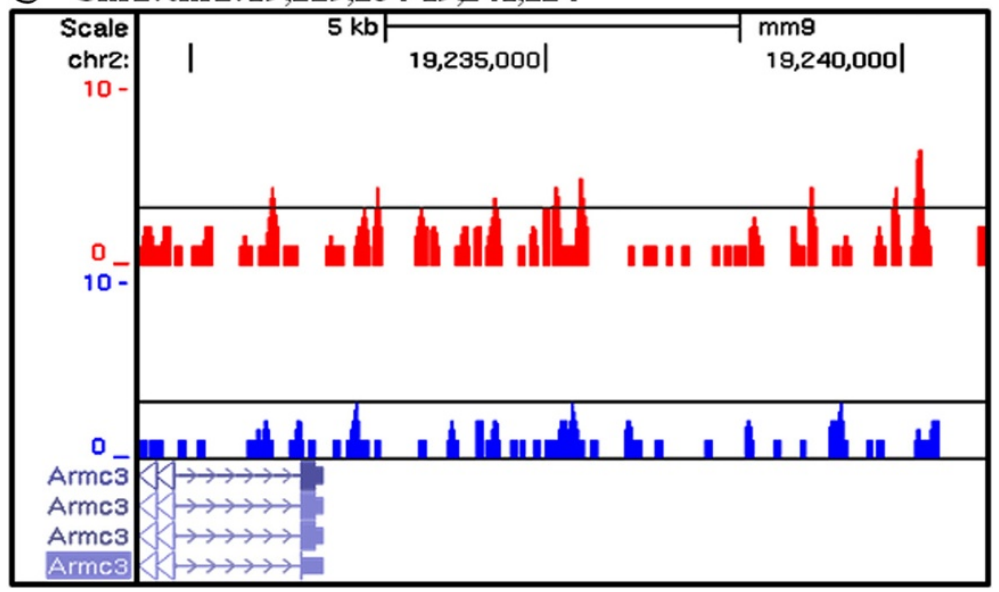

Figure 4 Examples of MeCP2 binding enrichment at specific gene regions. (A) MeCP2 binding at the TSS of Aard. (B) MeCP2 binding throughout the gene body of S/c38a1. (C) MeCP2 binding at the TES of Armc3. Cumulative DNA sequences co-purified with MeCP2 from wild-type astrocyte chromatin are depicted as wiggle tracks aligned to the UCSC genome browser NCBI37/mm9 track of the C57BL6/J mouse genome. Read counts are shown on the $\mathrm{Y}$ axis. MeCP2, methyl-CpG-binding protein 2; TES, transcriptional end site; TTS, transcriptional start sites. 
Table 1 qRT-PCR validation of expression microarray analyses for selected genes

\begin{tabular}{lllll}
\hline Gene & $\begin{array}{l}\text { Changes } \\
\text { according to } \\
\text { microarray }\end{array}$ & $\begin{array}{l}\text { Transcripts } \\
\text { significantly } \\
\text { increased } \\
\text { in -/y }\end{array}$ & $\begin{array}{l}\text { Transcripts } \\
\text { significantly } \\
\text { reduced } \\
\text { in -/y }\end{array}$ & $\begin{array}{l}\text { No significant } \\
\text { change } \\
\text { detected }\end{array}$ \\
\hline Slc38al & down in $-/ y$ & 1 & 0 & 3 \\
\hline Irx3 & down in $-/ y$ & 1 & 0 & 3 \\
\hline Ndn & down in $-/ y$ & 1 & 1 & 2 \\
\hline Nrep & down in $-/ y$ & 0 & 2 & 2 \\
\hline Lnpep & up in $-/ y$ & 1 & 0 & 3 \\
\hline Csrp & up in $-/ y$ & 2 & 0 & 2 \\
\hline Apoc2 & up in $-/ y$ & 3 & 0 & 1 \\
\hline Cdon & up in $-/ y$ & 3 & 0 & 1 \\
\hline Zmiz1 & up in $-/ y$ & 2 & 1 & 1 \\
\hline
\end{tabular}

enriched MeCP2 binding upstream of the TSS (Figure 5A). Similarly Slc38a1 and Armc3show enriched MeCP2 binding in the gene body and the TES respectively in qChIPPCR analysis (Figure $5 \mathrm{~B}$ and $5 \mathrm{C}$ ). These results show that subtle gene region differences in $\mathrm{MeCP} 2$ binding shown by ChIP-seq are reproduced in independent studies using alternative techniques.

cDNA samples from four biologic replicate astrocyte cultures were analyzed using primers designed to amplify transcripts from a chosen subset of genes with highly significant $(P<0.005)$ changes in transcript between wild-type and MeCP2-deficient astrocytes. Expression changes were normalized to Gapdh transcripts using the comparative Ct method. A one-tailed Student's $t$ test was used to identify significant changes $(P<0.05)$.

To confirm the integrated results of our combined astrocyte MeCP2 ChIP-seq and expression analysis, nine genes were selected for quantitative reverse transcriptase PCR (qRT-PCR) (Table 1). For these studies, astrocytes were cultured from four pairs of wild-type and Mecp2-deficient littermate pups. Total RNA was isolated from these cultures, reverse transcribed into cDNA and amplified using gene-specific primer pairs and primers to Gapdh in parallel. Comparative $\mathrm{Ct}$ analysis confirmed transcript alterations detected by microarray in three out of four biologic replicates for Cdon and $A p o c 2$, two out of four replicates for Csrp, Zmiz1 and Nrep (Table 1). Expression differences consistent with microarray data were detected in only one of four replicates for Slc38a, Irx3, Ndn and Lnpep, suggesting highly variable expression of these genes in astrocytes. Out of all the genes validated by qRT-PCR only Nrep had reduced expression in $\mathrm{MeCP} 2$-deficient mice while the other eight genes had elevated transcripts consistent with repression by $\mathrm{MeCP} 2$.

\section{Discussion}

The results presented above describe the first integrated, genome-wide screen for MeCP2 target genes in astrocytes.

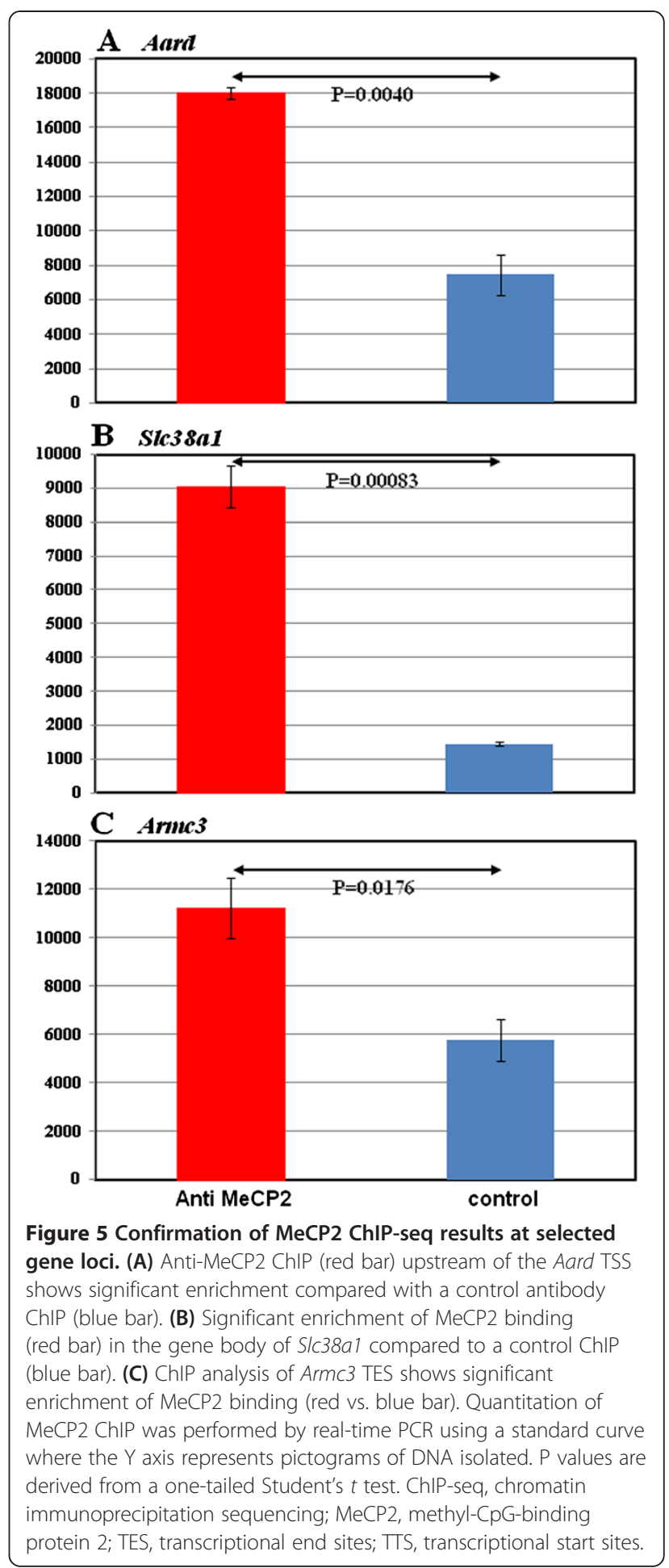

Previous expression microarray analyses have focused on identifying MeCP2 target genes in brain [8,10, , 26,27] or cell lines $[23,24,37]$ but not in individual cell types while MeCP2 ChIP-seq has been performed on sorted neuronal populations and astrocytes [11] but not in combination with expression analyses. To bridge this gap, the 118 genes 
identified in our stringent expression microarray analyses that were dysregulated by loss of MeCP2 (Table S2 in Additional file 1) were compared with 1973 genes having the highest levels of MeCP2 binding identified by independent ChIP-seq analyses. This combined analyses identified seventeen potential high-confidence MeCP2 target genes in astrocytes. Of these candidate targets, Aard1, Slc38a1 and Armc3 were independently validated for MeCP2 binding by qChIP-PCR (Figure 5).

Of these seventeen potential target genes, nine were selected for validation by qRT-PCR (Table 1). Functions of the validated genes were consistent with pathways affected in Rett syndrome. For example the glutamine transporter Slc38a1 (Snat1) is the rate-limiting transporter of glutamine across the plasma membrane and an important player in the glutamine-glutamate/GABA cycle for neurotransmitter generation and cycling [38], although the functional role of astrocytic glutamine transporters have been less frequently studied compared to their neuronal counterparts [39]. Irx3 encodes a factor critical for positioning of neuronal subtypes during neural tube differentiation [40]. Another novel, astrocytic MeCP2 target gene was maternally imprinted $N d n$. Ndn encodes Necdin, a growthsuppressing transcription factor, which may be deficient in the rare neurological disorder Prader-Willi syndrome [41]. Nrep, which has reduced transcripts in MeCP2 null astrocytes, is an important factor involved in glial mobility and neoplasia [42]. This may potentially contribute to observed glial defects in Mecp2-deficient mice [21,43]. Another target, Lnpep, encodes an insulin-regulated aminopeptidase, cleaves peptide hormones and may be involved in processing neuropeptides in the brain [44,45]. Csrp1, which has elevated astrocytic expression in the absence of $\mathrm{MeCP} 2$, appears to have a role in neuronal regeneration [46]. Defects in Cdon expression cause a severe structural defect in the developing brain [47]. Therefore this may contribute to Rett brain structure abnormalities. Although lipid abnormalities have not been reported, defects in the MeCP2 astrocyte target Apoc2 may contribute to cardiac defects reported in Rett model mice [48]. The last but not the least validated MeCP2 target in astrocytes, $\mathrm{Zmiz}$, was the sole gene to have the highest levels of MeCP2 binding in more than one gene region. Despite ubiquitous expression, Zmiz1, can potentiate the activity of the androgen receptor [49] and thereby affect a number of AR-responsive genes, including those in brain. Egrl, which was found to be a target of MeCP2 in neuronal cells [50], was tested but could not be validated by qRT-PCR for technical reasons. Due to the small number of total genes, DAVID only identified significant enrichment of MeCP2-responsive genes in the long-term depression pathway (Crh, Grial and Ppp2r2b) and genes in the cytokine-cytokine receptor pathway (Pdgfb, Il13ral and Osmr). It bears noting that like the Chahrour study [10] more genes (63) were downregulated by loss of MeCP2 than were upregulated (55) suggesting that MeCP2 can activate as well as repress gene expression.

The remaining 108 genes identified in our expression microarray screen indicate that a restricted subset of astrocyte function is impaired by loss of MeCP2 directly or indirectly. For example, Ier2 (immediate early response 2), Egr1, Fos, and Jun that function as immediate, early transcription factors are dysregulated. Altered expression of any of these factors alone could affect transcription of multiple genes responding to sensory input. Other affected genes like Cntn1 (Contactin 1), Syn2 (Synapsin 2), Gabrg1, and Gria1 function at the synapse where astrocytes make key functional crosstalk with neurons in a structural unit called a tripartite synapse [51]. Dysregulation of these genes in astrocytes, therefore, could affect the ability of astrocytes to sense neuronal activity and to modulate synaptic transmission. Interestingly, the immune adhesion molecules Icam1 (intercellular adhesion molecule 1), Itga1 (integrin alpha 1), Msr1 (macrophage scavenger receptor 1), and the complement pore-forming protein-coding genes $\mathrm{C1} r, \mathrm{C} 3$, along with the lysozyme-encoding gene $L y z 2$, which are key regulators of the innate immune system, are significantly affected. This is consistent with recent studies showing a key role for glia immune effector pathways in a mouse model of Rett syndrome [52]. Also there is emerging evidence that factors with immune function also have neurologic function in the immune system [53]. Genes in the insulin signaling pathway, Ide, Igfbp4 (insulin-like growth factor binding protein 4) also show dysregulation in astrocytes. This finding is consistent with reports of insulin pathway defects in Rett syndrome [54] [55]. The current study also reveals that expression of the solute carrier genes, Slc10a3, Slc11a1, Slc16a13, Slc38a1 are dysregulated. Further analyses of glutamate pathway defects in MeCP2-deficient astrocytes are being pursued.

Overall there are some interesting conclusions that can be drawn from the current study. Together, these expression profiling and ChIP results suggest that astrocytes have a unique set of MeCP2 target genes. For example while $I d 1, I d 2, I d 3$ and $I d 4$ genes encode transcription factors [24] that are preferentially expressed in astrocytes [56], they do not appear to be targets of MeCP2 but are targets in neuronal cells [26]. Although astrocytes compose the majority of cells in the brain, MeCP2 target genes in astrocytes may have been overlooked in previous studies. Expression profiling of whole hypothalami with increased or diminished levels of MeCP2 revealed that 2582 genes were dysregulated at $P<0.05$ [10]. Of those genes, only Crh, Gabrg1, Pdgfb and Ror1 (receptor tyrosine kinase-like orphan receptor 1 ) were found to be dysregulated $(P<0.005)$ in our screen. Again, as neurons in the hypothalamus and brain have the highest levels of MeCP2 [11], this result is not unexpected. Further studies on this unique set of MeCP2 target genes in astrocytes 
could help us to understand why wild-type glia are able to rescue aspects of the Rett phenotype in mouse models [22] and offer opportunities for novel therapies.

\section{Conclusions}

In summary, these studies have successfully identified a unique set of genes responsive to MeCP2 in astrocytes. As it has been shown that wild-type astrocytes can restore some function to $\mathrm{MeCP} 2$-deficient mice these findings have the potential to advance new therapeutic interventions targeting astrocytes for the treatment of Rett syndrome.

\section{Availability of supporting data}

The data sets supporting the results of this article are available in the NIH Gene Expression Omnibus (GEO) repository (www.ncbi.nlm.nih.gov/geo).

\section{Additional file}

Additional file 1: Table S1. Primers used for RT-PCR. Table S2. RNA transcripts altered by loss of MeCP2 $(P<0.0005)$. Table S3. Primers used for ChIP-seq validation.

\section{Abbreviations}

MeCP2: Methyl-CpG-binding protein 2; ChIP-seq: Chromatin immunoprecipitation sequencing; FDR: False discovery rate; qRTPCR: Quantitative reverse transcriptase polymerase chain reaction; RPKM: Reads per kilobase of element assayed per million mapped reads; RTT: Rett syndrome; TES: Transcription end site; TSS: Transcription start site; qChIP-PCR: Quantitative chromatin immunoprecipitation polymerase chain reaction.

\section{Competing interests}

The authors have no competing interests to declare.

\section{Authors' contributions}

$\mathrm{DHY}$ carried out the MeCP2 ChIP-seq studies and wrote the manuscript. HX performed expression microarray profiling and analysis. KWD carried out the ChIP-seq analysis and performed RT-PCR validation of microarray data. LWJ participated in the experimental design and analysis. JML participated in the design of the study and was consulted on data analyses. IM conceived of the study, generated primary astrocyte cultures and participated in its design and coordination. All authors read and approved the final manuscript.

\section{Acknowledgements}

The authors would like to acknowledge Frank R. Sharp, Ryan R. Davis, Haley A. Scoles, Diane I. Schroeder and Leath Tonkin for advice and data acquisition. This manuscript was supported by NIH awards to JML (R01 HD048799, 2R01HD041462, and R01 NS076263), LWJ (R01 HD064817), and IM (R21 HD073631).

\section{Author details \\ 1Department of Medical Microbiology and Immunology, UC Davis Genome Center, University of California Davis, 1 Shields Avenue, Davis, CA 95616, USA. ${ }^{2}$ University of California Davis, MIND Institute, 2825 50th Street, Sacramento, CA 95817, USA. ${ }^{3}$ Center for Research on Genomics and Global Health, National Human Genome Research Institute, National Institutes of Health, 12 South Drive, Bethesda, MD 20892, USA. ${ }^{4}$ Department of Pathology and Laboratory Medicine, University of California Davis Medical Center, $4400 \mathrm{~V}$ Street, Sacramento, CA 95817, USA.}

Received: 20 September 2012 Accepted: 10 January 2013 Published: 25 January 2013

\section{References}

1. Chahrour M, Zoghbi HY: The story of Rett syndrome: from clinic to neurobiology. Neuron 2007, 56:422-437

2. Hagberg B, Aicardi J, Dias K, Ramos O: A progressive syndrome of autism, dementia, ataxia, and loss of purposeful hand use in girls: Rett's syndrome: report of 35 cases. Ann Neurol 1983, 14:471-479.

3. Amir RE, Van den Veyver IB, Wan M, Tran CQ, Francke U, Zoghbi HY: Rett syndrome is caused by mutations in X-linked $M E C P 2$, encoding methyl- CpG-binding protein 2. Nat Genet 1999, 23:185-188.

4. Mari F, Azimonti S, Bertani I, Bolognese F, Colombo E, Caselli R, Scala E, Longo I, Grosso S, Pescucci C, Ariani F, Hayek G, Balestri P, Bergo A, Badaracco G, Zappella M, Broccoli V, Renieri A, Kilstrup-Nielsen C, Landsberger N: CDKL5 belongs to the same molecular pathway of $\mathrm{MeCP} 2$ and it is responsible for the early-onset seizure variant of Rett syndrome. Hum Mol Genet 2005, 14:1935-1946.

5. Scala E, Ariani F, Mari F, Caselli R, Pescucci C, Longo I, Meloni I, Giachino D, Bruttini M, Hayek G, Zappella M, Renieri A: CDKL5/STK9 is mutated in Rett syndrome variant with infantile spasms. J Med Genet 2005, 42:103-107.

6. Ariani F, Hayek G, Rondinella D, Artuso R, Mencarelli MA, Spanhol-Rosseto A, Pollazzon M, Buoni S, Spiga O, Ricciardi S, Meloni I, Longo I, Mari F, Broccoli V, Zappella M, Renieri A: FOXG1 is responsible for the congenital variant of Rett syndrome. Am J Hum Genet 2008, 83:89-93.

7. Jones PL, Veenstra GJ, Wade PA, Vermaak D, Kass SU, Landsberger N, Strouboulis J, Wolffe AP: Methylated DNA and MeCP2 recruit histone deacetylase to repress transcription. Nat Genet 1998, 19:187-191.

8. Tudor M, Akbarian S, Chen RZ, Jaenisch R: Transcriptional profiling of a mouse model for Rett syndrome reveals subtle transcriptional changes in the brain. Proc Natl Acad Sci USA 2002, 99:15536-15541.

9. Yasui DH, Peddada S, Bieda MC, Vallero RO, Hogart A, Nagarajan RP, Thatcher KN, Farnham PJ, Lasalle JM: Integrated epigenomic analyses of neuronal $\mathrm{MeCP} 2$ reveal a role for long-range interaction with active genes. Proc Natl Acad Sci USA 2007, 104:19416-19421.

10. Chahrour M, Jung SY, Shaw C, Zhou X, Wong ST, Qin J, Zoghbi HY: MeCP2, a key contributor to neurological disease, activates and represses transcription. Science 2008, 320:1224-1229.

11. Skene PJ, Illingworth RS, Webb S, Kerr AR, James KD, Turner DJ, Andrews R, Bird AP: Neuronal MeCP2 is expressed at near histone-octamer levels and globally alters the chromatin state. Mo/ Cell 2010, 37:457-468.

12. Cohen S, Gabel HW, Hemberg M, Hutchinson AN, Sadacca LA, Ebert DH, Harmin DA, Greenberg RS, Verdine VK, Zhou Z, Wetsel WC, West AE, Greenberg ME: Genome-wide activity-dependent MeCP2 phosphorylation regulates nervous system development and function. Neuron 2011, 72:72-85.

13. Horike S, Cai S, Miyano M, Cheng JF, Kohwi-Shigematsu T: Loss of silent-chromatin looping and impaired imprinting of DLX5 in Rett syndrome. Nat Genet 2005, 37:31-40.

14. Yasui DH, Scoles HA, Horike S, Meguro-Horike M, Dunaway KW, Schroeder DI, Lasalle JM: 15q11.2-13.3 chromatin analysis reveals epigenetic regulation of CHRNA7 with deficiencies in Rett and autism brain. Hum Mol Genet 2011, 20:4311-4323.

15. Nikitina T, Ghosh RP, Horowitz-Scherer RA, Hansen JC, Grigoryev SA, Woodcock CL: MeCP2-chromatin interactions include the formation of chromatosome-like structures and are altered in mutations causing Rett syndrome. J Biol Chem 2007, 282:28237-28245.

16. Young Jl, Hong EP, Castle JC, Crespo-Barreto J, Bowman AB, Rose MF, Kang D, Richman R, Johnson JM, Berget S, Zoghbi HY: Regulation of RNA splicing by the methylation-dependent transcriptional repressor methyl-CpG binding protein 2. Proc Natl Acad Sci USA 2005, 102:17551-17558.

17. Guy J, Hendrich B, Holmes M, Martin JE, Bird A: A mouse Mecp2-null mutation causes neurological symptoms that mimic Rett syndrome. Nat Genet 2001, 27:322-326.

18. Chen RZ, Akbarian S, Tudor M, Jaenisch R: Deficiency of methyl-CpG binding protein-2 in CNS neurons results in a Rett-like phenotype in mice. Nat Genet 2001, 27:327-331.

19. Chen WG, Chang Q, Lin Y, Meissner A, West AE, Griffith EC, Jaenisch R, Greenberg ME: Derepression of BDNF transcription involves calciumdependent phosphorylation of MeCP2. Science 2003, 302:885-889.

20. Zhou Z, Hong EJ, Cohen S, Zhao WN, Ho HY, Schmidt L, Chen WG, Lin Y, Savner E, Griffith EC, Hu L, Steen JA, Weitz CJ, Greenberg ME: Brain-specific phosphorylation of MeCP2 regulates activity-dependent Bdnf 
transcription, dendritic growth, and spine maturation. Neuron 2006, 52:255-269.

21. Maezawa I, Swanberg S, Harvey D, LaSalle JM, Jin LW: Rett syndrome astrocytes are abnormal and spread MeCP2 deficiency through gap junctions. J Neurosci 2009, 29:5051-5061.

22. Lioy DT, Garg SK, Monaghan CE, Raber J, Foust KD, Kaspar BK, Hirrlinger PG, Kirchhoff F, Bissonnette JM, Ballas N, Mandel G: A role for glia in the progression of Rett's syndrome. Nature 2011, 475:497-500.

23. Traynor J, Agarwal P, Lazzeroni L, Francke U: Gene expression patterns vary in clonal cell cultures from Rett syndrome females with eight different MECP2 mutations. BMC Med Genet 2002, 3:12.

24. Peddada S, Yasui DH, LaSalle JM: Inhibitors of differentiation (ID1, ID2, ID3 and ID4) genes are neuronal targets of MeCP2 that are elevated in Rett syndrome. Hum Mol Genet 2006, 15:2003-2014.

25. Kriaucionis S, Paterson A, Curtis J, Guy J, Macleod N, Bird A: Gene expression analysis exposes mitochondrial abnormalities in a mouse model of Rett syndrome. Mol Cell Biol 2006, 26:5033-5042

26. Jordan C, Li HH, Kwan HC, Francke U: Cerebellar gene expression profiles of mouse models for Rett syndrome reveal novel MeCP2 targets. BMC Med Genet 2007, 8:36.

27. Gibson JH, Slobedman B, NH K, Williamson SL, Minchenko D, El-Osta A Stern JL, Christodoulou J: Downstream targets of methyl CpG binding protein 2 and their abnormal expression in the frontal cortex of the human Rett syndrome brain. BMC Neurosci 2010, 11:53.

28. Maezawa I, Nivison M, Montine KS, Maeda N, Montine TJ: Neurotoxicity from innate immune response is greatest with targeted replacement of E4 allele of apolipoprotein $\mathrm{E}$ gene and is mediated by microglial p38MAPK. FASEB J 2006, 20:797-799.

29. Wu Z, Irizarry RA, Gentleman R, Martinez-Murillo F, Spencer F: A modelbased background adjustment for oligonucleotide expression arrays. J Am Stat Assoc 2004, 99:909-917.

30. Reiner A, Yekutieli D, Benjamini Y: Identifying differentially expressed genes using false discovery rate controlling procedures. Bioinformatics 2003, 19:368-375.

31. Dennis G Jr, Sherman BT, Hosack DA, Yang J, Gao W, Lane HC, Lempicki RA: DAVID: Database for Annotation, Visualization, and Integrated Discovery. Genome Biol 2003, 4:P3.

32. Hosack DA, Dennis G Jr, Sherman BT, Lane HC, Lempicki RA: Identifying biological themes within lists of genes with EASE. Genome Bio/ 2003, 4:R70,

33. Blahnik KR, Dou L, O'Geen H, McPhillips T, Xu X, Cao AR, lyengar S, Nicolet CM, Ludascher B, Korf I, Farnham PJ: Sole-Search: an integrated analysis program for peak detection and functional annotation using ChIP-seq data. Nucleic Acids Res 2010, 38:e13.

34. Hon G, Ren B, Wang W: ChromaSig: a probabilistic approach to finding common chromatin signatures in the human genome. PLoS Comput Biol 2008, 4:e1000201.

35. Zhang Y, Liu T, Meyer CA, Eeckhoute J, Johnson DS, Bernstein BE, Nusbaum C, Myers RM, Brown M, Li W, Liu XS: Model-based analysis of ChIP-Seq (MACS). Genome Biol 2008, 9:R137.

36. Cahoy JD, Emery B, Kaushal A, Foo LC, Zamanian JL, Christopherson KS, Xing Y, Lubischer JL, Krieg PA, Krupenko SA, Thompson WJ, Barres BA: A transcriptome database for astrocytes, neurons, and oligodendrocytes: a new resource for understanding brain development and function. J Neurosci 2008, 28:264-278.

37. Delgado IJ, Kim DS, Thatcher KN, LaSalle JM, Van den Veyver IB: Expression profiling of clonal lymphocyte cell cultures from Rett syndrome patients. BMC Med Genet 2006, 7:61.

38. Mackenzie B, Erickson JD: Sodium-coupled neutral amino acid (System N/ A) transporters of the SLC38 gene family. Pflugers Arch 2004, 447:784-795.

39. Melone M, Quagliano F, Barbaresi P, Varoqui H, Erickson JD, Conti F: Localization of the glutamine transporter SNAT1 in rat cerebral cortex and neighboring structures, with a note on its localization in human cortex. Cereb Cortex 2004, 14:562-574.

40. Briscoe J, Pierani A, Jessell TM, Ericson J: A homeodomain protein code specifies progenitor cell identity and neuronal fate in the ventral neural tube. Cell 2000, 101:435-445.

41. Jay P, Rougeulle C, Massacrier A, Moncla A, Mattei MG, Malzac P, Roeckel N, Taviaux S, Lefranc JL, Cau P, Berta P, Lalande M, Muscatelli F: The human necdin gene, NDN, is maternally imprinted and located in the Prader-Willi syndrome chromosomal region. Nat Genet 1997, 17:357-361.
42. McDonough WS, Tran NL, Berens ME: Regulation of glioma cell migration by serine-phosphorylated P311. Neoplasia 2005, 7:862-872.

43. Ballas N, Lioy DT, Grunseich C, Mandel G: Non-cell autonomous influence of MeCP2-deficient glia on neuronal dendritic morphology. Nat NeurosC 2009, 12:311-317.

44. Matsumoto A, Motozaki K, Seki T, Sasaki R, Kawabe T: Expression of human brain carboxypeptidase $B$, a possible cleaving enzyme for beta-amyloid precursor protein, in peripheral fluids. Neurosci Res 2001, 39:313-317.

45. Matsumoto H, Nagasaka T, Hattori A, Rogi T, Tsuruoka N, Mizutani S, Tsujimoto M: Expression of placental leucine aminopeptidase/ oxytocinase in neuronal cells and its action on neuronal peptides. Eur J Biochem 2001, 268:3259-3266.

46. Ma L, Yu YM, Guo Y, Hart RP, Schachner M: Cysteine- and glycine-rich protein 1a is involved in spinal cord regeneration in adult zebrafish. Eur J Neurosci 2012, 35:353-365.

47. Bae GU, Domene S, Roessler E, Schachter K, Kang JS, Muenke M, Krauss RS: Mutations in $\mathrm{CDON}$, encoding a hedgehog receptor, result in holoprosencephaly and defective interactions with other hedgehog receptors. Am J Hum Genet 2011, 89:231-240.

48. Seyfried TN, Heinecke KA, Mantis JG, Denny CA: Brain lipid analysis in mice with Rett syndrome. Neurochem Res 2009, 34:1057-1065.

49. Sharma M, Li X, Wang Y, Zarnegar M, Huang CY, Palvimo JJ, Lim B, Sun Z: hZimp10 is an androgen receptor co-activator and forms a complex with SUMO-1 at replication foci. EMBO J 2003, 22:6101-6114.

50. Swanberg SE, Nagarajan RP, Peddada S, Yasui DH, LaSalle JM: Reciprocal co-regulation of EGR2 and MECP2 is disrupted in Rett syndrome and autism. Hum Mol Genet 2009, 18:525-534.

51. Santello M, Cali C, Bezzi P: Gliotransmission and the tripartite synapse. Adv Exp Med Biol 2012, 970:307-331.

52. Derecki NC, Cronk JC, Lu Z, Xu E, Abbott SB, Guyenet PG, Kipnis J: Wildtype microglia arrest pathology in a mouse model of Rett syndrome. Nature 2012, 484:105-109.

53. Boulanger LM, Shatz CJ: Immune signalling in neural development, synaptic plasticity and disease. Nat Rev Neurosci 2004, 5:521-531.

54. Itoh M, Ide S, Takashima S, Kudo S, Nomura Y, Segawa M, Kubota T, Mori H, Tanaka S, Horie H, Tanabe Y, Goto Y: Methyl CpG-binding protein 2 (a mutation of which causes Rett syndrome) directly regulates insulinlike growth factor binding protein 3 in mouse and human brains. J Neuropathol Exp Neurol 2007, 66:117-123.

55. Tropea D, Giacometti E, Wilson NR, Beard C, McCurry C, Fu DD, Flannery R, Jaenisch R, Sur M: Partial reversal of Rett Syndrome-like symptoms in MeCP2 mutant mice. Proc Natl Acad Sci USA 2009, 106:2029-2034.

56. Kitamura $M$, Itoh $K$, Matsumoto $A$, Hayashi $Y$, Sasaki $R$, Imai $Y$, Itoh $H$ : Prenatal ionizing radiation-induced apoptosis of the developing murine brain with special references to the expression of some proteins. Kobe $J$ Med Sci 2001, 47:59-76.

doi:10.1186/2040-2392-4-3

Cite this article as: Yasui et al: MeCP2 modulates gene expression pathways in astrocytes. Molecular Autism 2013 4:3.

\section{Submit your next manuscript to BioMed Central and take full advantage of:}

- Convenient online submission

- Thorough peer review

- No space constraints or color figure charges

- Immediate publication on acceptance

- Inclusion in PubMed, CAS, Scopus and Google Scholar

- Research which is freely available for redistribution 